\title{
ON THE ABSENCE OF ANTIMATTER FROM THE UNIVERSE, DARK MATTER, THE FINE STRUCTURE PARAMETER, THE FLUCTUATIONS OF THE COSMIC MICROWAVE BACKGROUND RADIATION AND THE TEMPERATURE DIFFERENCE BETWEEN THE NORTHERN AND SOUTHERN HEMISPHERE OF THE UNIVERSE
}

\author{
Emmanuil Manousos \\ Department of Physics, Astrophysics Laboratory, National and Kapodistrian University of Athens, \\ Panepistimiopolis, GR 15783 Zographos, Athens, Greece
}

Received 2014-02-05; Revised 2014-03-17; Accepted 2014-3-21

\begin{abstract}
The theory of selfvariations correlates five cosmological observations considered to be unrelated by the physical theories of the previous century. The absence of antimatter from the Universe, the Dark Matter, the slight variation of the fine structure parameter, the temperature fluctuations of the cosmic microwave background radiation and the temperature difference between the northern and southern hemisphere of the Universe can be justified by a common cause. This cause is the selfvariation of the electric charge of material particles. The antimatter particles of the very early Universe lose their electric charge with the passage of time and end up as electrically neutral. These electrically neutral particles constitute a significant part of Dark Matter. The cosmological Model of the Selfvariations predicts another possible mechanism for the creation of Dark Matter particles. Thus, we can justify the fact that the amount of Dark Matter is greater than the amount of the ordinary, luminous, matter. A fluctuation of the electric charge at cosmological distances is predicted in the region of the Universe that we observe. This fluctuation is recorded in the cosmological data in the value of the fine structure parameter measured at cosmological distances, in the temperature of the cosmic microwave background radiation and is responsible for the temperature difference between the two hemispheres of the Universe. The study we present proves in detail that the law of selfvariations contains enough information to justify the totality of cosmological data that cannot be justified by the standard cosmological model. These data have been observed by the ultrasensitive modern observation instruments. The high sensitivity of the instruments is necessary to record the effects from the extremely small variation of the electric charge. We regard as necessary a re-evaluation of the cosmological data based on the law of selfvariations.
\end{abstract}

Keywords: Antimatter, Dark Matter, Fine Structure Parameter, CMBR, Oklo

\section{INTRODUCTION}

The law of selfvariations expresses quantitatively a slight continuous increase of the rest mass and the electric charge of material particles. In the macrocosm, the law of selfvariations is expressed by a simple differential equation for the rest mass and by a similar one for the electric charge of material particles. The solutions resulting from these differential equations justify the totality of the cosmological data. Some cosmological data, like the redshift of distant astronomical objects, the Cosmic Microwave Background Radiation (CMBR), the nucleosynthesis of chemical elements and the increased luminosity distances of Type Ia supernovae, result mainly as a consequence of the selfvariation of the rest mass. The selfvariation of the electric charge is respensible for large part of the Dark 
Matter. The absence of antimatter from the Universe, the fluctuation of the fine structure parameter observed at cosmological distances, the temperature fluctuation of the CMBR and the temperature difference between the two hemispheres of the Universe are exclusively due to the selfvariation of the electric charge.

In the microcosm, the law of selfvariations predicts that the rest mass and the electric charge of material particles spread, are distributed, within spacetime. When we try to define this distribution, the Schrödinger equation, as well as the relevant equations, appear and play a fundamental role. These equations for the microcosm, replace the simple differential equation given by the law of selfvariations for the macrocosm.

The selfvariation of the rest mass evolves only in the direction of increase of the rest masses of material particles. On the contrary, the selfvariation of the magnitude of the electric charge can evolve in two different directions. The electric charge of material particles can either increase, or decrease, in absolute value. This difference arises from the fact that the electric charge exists in the Universe as pairs of opposite quantities. In this article we examine in detail the evolution of the selfvariation of the electric charge in two directions and its consequences.

\section{FUNDAMENTAL EQUATIONS OF THE COSMOLOGICAL MODEL OF THE SELFVARIATIONS}

The law of selfvariations in the macrocosm predicts (Manousos, 2013a, Equation 270 and 292) Equation:

$$
\left(m_{0}+\frac{i \hbar}{c^{2}} \frac{\dot{m}_{0}}{m_{0}}\right)^{\cdot}=0
$$

For the rest mass $\mathrm{m}_{0}$ of material particles and the corresponding Equation:

$$
\left(q+\frac{i \hbar}{V_{0}} \frac{\dot{q}}{q}\right)^{\cdot}=0
$$

For the magnitude $q(q>0)$ of the electric charge of material particles. In Equation 2 the electromagnetic potential $V_{O}$ is independent of the selfvariations (Manousos, 2013b). With $(\bullet)$ we denote the derivative with respect to time $t$. Equation 1 and 2 are solved in a flat and static Universe (Manousos, 2013a).

Solving Equation 1 we find relation:

$$
m_{0}(r)=m_{0} \frac{1-A}{1-A \exp \left(-\frac{k r}{c}\right)}
$$

Between the rest mass $m_{0}(r)$ of a material particle in a distant astronomical object at distance $r$ from Earth and the laboratory value of the rest mass $m_{0}$ of the same particle on earth. Between parameters $k$ and $A$, the following relation holds:

$$
\frac{k A}{1-A}=H
$$

where, $H$ is Hubble's parameter. Parameter $A$ increases very slightly with the passage of time $t$ according to Equation 5:

$\frac{d A}{d t}=\dot{A}=k A$

While it obeys inequality:

$\frac{z}{1+z}<A<1$

For every value of the redshift $z$.

Solving Equation 2 we similarly obtain:

$$
q(r)=q \frac{1-B}{1-B \exp \left(-\frac{k_{1} r}{c}\right)}
$$

$\frac{d B}{d t}=\dot{B}=k_{1} B$

Given the fact that we know the value of Hubble's parameter $H$, Equation 4 provides a relation between parameters $k$ and $A$. Furthermore, Equation 6 confines to a satisfactory degree the values parameter $A$ can take. Thus, we were able to derive a large amount of information about the consequences of the selfvariation of the rest mass at cosmological scales (Manousos, 2013a; 2013b).

Regarding the selfvariation of the electric charge, we know that $B>0$ and that it evolves at an extremely slow rate (Manousos, 2013b). We shall now repeat the proof of Equation 7 from Equation 2 in order to highlight the fundamental parameters defining the selfvariation of the electric charge. 
From Equation 2 we obtain:

$q+\frac{i \hbar}{V_{0}} \frac{\dot{q}}{q}=\sigma_{1}$

where, $\sigma_{1}$ is the integration constant, measured in units of electric charge. By denoting Equation 10:

$x=\frac{q}{\sigma_{1}}$

Equation 9 can be written as:

$x+\frac{i \hbar}{V_{0} \sigma_{1}} \frac{\dot{x}}{x}=1 \frac{i \hbar}{V_{0} \sigma_{1}} \frac{\dot{x}}{x}=1-x$

$\dot{x}=\frac{i V_{0} \sigma_{1}}{\hbar} x(x-1) \frac{d x}{x(1-x)}=\frac{i V_{0} \sigma_{1}}{\hbar} d t$

$\left(\frac{1}{x-1}-\frac{1}{x}\right) d x=\frac{i V_{0} \sigma_{1}}{\hbar} d t$

We integrate Equation 11 between moment $t_{0}$, when the electric charge has value $q_{0}$ and $x=\frac{q_{0}}{\sigma_{1}}$ and moment $t$, "now", when the electric charge has value $\mathrm{q}$ and $x=\frac{q}{\sigma_{1}}$ and after performing the calculations, we get:

$$
q=\frac{\sigma_{1}}{1-\frac{q_{0}-\sigma_{1}}{q_{0}} \exp \left(\frac{i V_{0} \sigma_{1}}{\hbar}\left(t-t_{0}\right)\right)}
$$

In order to find the value of the electric charge $q(r)$ at a distant astronomical object located at distance $r$ from Earth, we replace $t$ in Equation 12 with $t-\frac{r}{c}$ and get:

$$
q(r)=\frac{\sigma_{1}}{1-\frac{q_{0}-\sigma_{1}}{q_{0}} \exp \left(\frac{i V_{00} \sigma_{1}}{\hbar}\left(t-t_{0}\right)\right) \cdot \exp \left(-\frac{i V_{0} \sigma_{1}}{\hbar} \frac{r}{c}\right)}
$$

Denoting Equation 14:

$\mathrm{B}=\frac{q_{0}-\sigma_{1}}{q_{0}} \exp \left(\frac{i V_{0} \sigma_{1}}{\hbar}\left(t-t_{0}\right)\right)=\frac{q_{0}-\sigma_{1}}{q_{0}} \exp \left(k_{1}\left(t-t_{0}\right)\right)$

$k_{1}=\frac{i V_{0} \sigma_{1}}{\hbar}$
Equation 12 and 13 can be written correspondingly as:

$$
q=\frac{\sigma_{1}}{1-B} q(r)=\frac{\sigma_{1}}{1-B \exp \left(-\frac{k_{1} r}{c}\right)}
$$

From which we obtain:

$$
q(r)=q \frac{1-B}{1-B \exp \left(-\frac{k_{1} r}{c}\right)}
$$

Which is Equation 7. Similarly, Equation 8 results directly from Equation 14.

In the law of selfvariations (Manousos, 2013a, Equation 265 and 266), the imaginary unit $i$ has being introduced in order to incorporate into the statement of the law the consequences stemming from the internality of the Universe in the process of measurement (Manousos, 2013b). The final Equation 3 and 7, i.e., the solutions of Equation 1 and 2, do not change if we replace the imaginary unit with any constant $b \neq 0$ in Equation 1 and 2 (Manousos, 2013a). In the macrocosm we measure the consequences of a real variation of the rest masses and the electric charges of material particles, something that cannot be done in the microcosm (Manousos, 2013b). We could reformulate the law of selfvariations by initially assigning any arbitrary parameter $b \neq 0$ in the place of $i$.

In order to avoid the confusion that might arise from the presence of the imaginary unit $i$, we write Equation 15 as:

$k_{1}=\frac{b V_{0} \sigma_{1}}{\hbar}$

As we shall see, the arbitrary parameter $b \neq 0$ does not play any role in the resulting conclusions, since they are determined by the value of parameter $k_{l}$. This is the parameter we can measure on the basis of the cosmological data. The variation of the electric charge $q$ results in the variation of the fine structure parameter $\alpha$ Equation 17:

$\alpha=\frac{q^{2}}{4 \pi \varepsilon_{0} c \hbar}$

From the very slight variation of parameter $\alpha$ for cosmological distances (Webb et al., 2011; King et al., 2011; Molaro et al., 2008; Murphy et al., 2008; 2007; 
Tzanavaris et al., 2005; Chand et al., 2004; Murphy et al., 2003; Webb et al., 2001; Dzuba et al., 1999; Webb et al., 1999), we conclude that the selfvariation of the electric charge evolves at an extremely slow rate.

\section{THE PARTICLES OF THE ELECTRICALLY CHARGED ANTIMATTER OF THE EARLY UNIVERSE ARE CONVERTED, WITH THE PASSAGE OF TIME, INTO DARK MATTER PARTICLES}

By comparing Equation 1 and 2, we find that in place of the electromagnetic potential $V_{0}$ in Equation 2, factor $c^{2}>0$ appears in Equation 1. This is a general characteristic of the Equations resulting from the law of selfvariations and appears from the begining in the Equations of the theory of selfvariations (Manousos, 2013a, see: Energymomentum tensors 254,259 and the remark in paragraph 4.8). The fact that $c^{2}>0$ has as a consequence that the selfvariation of the rest mass occurs in the direction of increase of the rest mass of material particles. There are also other arguments that strengthen this conclusion, which we will not mention in the present article.

The electromagnetic potential $V_{0}$ can be either positive $\left(V_{0}>0\right)$ or negative $\left(V_{0}<0\right)$. According to Equation 16 , a change of sign of the electromagnetic potential $V_{0}$ brings about a change of sign of parameter $k_{l}$. This causes parameter $k_{1}$ to be either positive $\left(k_{l}>0\right)$ or negative $\left(k_{1}<0\right)$. But, according to Equation 7, for $k_{1}>0$ the selfvariation of the electric charge evolves in the direction of increase of the electric charge in absolute value, whereas for $\mathrm{k}_{1}<0$ the selfvariation evolves in the direction of decrease of the electric charge in absolute value. Consequently, the possibility for the electromagnetic potential $V_{0}$ to be either positive or negative is the reason why the selfvariation of the electric charge can evolve in two directions.

The electric charge has an initial value $q_{0}$ at moment $\mathrm{t}_{0}$ in the distant past, in the very early Universe. In the case where $k_{1}>0$ the electric charge increases in absolute value, at an extremely slow rate and reaches the value we measure in the laboratory today. But in the case where $k_{l}<0$, the electric charge decreases in absolute value. If this happens for a long enough time, the electric charge tends to vanish and the initially charged particles are electrically neutral today.

We will now determine a difference between the atoms of matter and the atoms of antimatter, which could justify the change in the sign of the electromagnetic potential $V_{O}$ of Equation 16 between the two kinds of atoms. In the case of matter, in the hydrogen atom the negative electric charge of the electron overlaps the positive electric charge of the proton. In the case of antimatter, the positive electric charge of the positron overlaps the negative electric charge of the antiproton. This reversal of the sign of the electric charge could justify the change in sign of the electromagnetic potential $V_{O}$ at the moment when the opposite electric charges appear. In the macrocosm we know that the electromagnetic potential created by two opposite electric charges changes sign, at every point in space, if we reverse the sign of the two electric charges. Of course, in the case we are studying, further investigation is required, something natural since the investigation of the law of selfvariations is at its initial stage. Nevertheless, the possibility of conversion of the antimatter particles of the very early Universe into electrically neutral particles, is a clear prediction of the theory of selfvariations.

The change of sign of potential $V_{0}$ between matter and antimatter can justify in a unified way, with a common cause, the absence of antimatter in the Universe today and the origin of a large number of Dark Matter particles. For the potential $V_{0}$ for which parameter $k_{l}$ in Equation 16 is positive $\left(k_{l}>0\right)$, the electric charge of particles increases with the passage of time. This leads to the hydrogen atom, as we observe it today. For the potential $V_{0}$ for which parameter $\mathrm{k}_{1}$ in Equation 16 is negative $\left(k_{l}<0\right)$, the initially electrically charged particles loose their electric charge with the passage of time. According to the difference we specified between matter and antimatter regarding the sign of potential $V_{0}$, the antimatter particles loose their electric charge with the passage of time and end up electrically neutral. These particles, without electric charge, behave like Dark Matter particles.

If initially there were equal quantities of matter and antimatter particles in the Universe, $50 \%$ of the particles loose their electric charge and, with the passage of time, are converted into Dark Matter particles. The cosmological model of the selfvariations predicts further reasons favoring the creation of Dark Matter particles (Manousos, 2013a; 2013b). Thus, the large amount of Dark Matter recorded in the cosmological data, can be justified.

A resulting indirect conclusion is that the antimatter particles loose their electric charge before the accumulation of matter for the formation of the large structures in the Universe. This conclusion arises from the fact that antimatter is absent from the large-scale structures of the Universe we observe today. Due to the extremely slow rate of evolution of the selfvariation of 
the electric charge, a very long time is required for the antimatter particles to loose their charge. Therefore, a very long time is required for the Universe to evolve from its initial state into the state we observe today. This is consistent with the prediction of the model of selfvariations about the age and size of the Universe (Manousos, 2013a; 2013b). We note that the cosmological model of the selfvariations is selfconsistent and its predictions should be correlated, where necessary, with the initial form of the Universe predicted by the model itself and not with the initial form of the Universe predicted by other models.

\section{ON THE VARIATION OF THE FINE STRUCTURE PARAMETER $\alpha$}

Due to the very large age of the Universe, every material particle has its own past history and it is possible for external factors to act additively and bring about a slight fluctuation in the value of the electromagnetic potential $V_{0}$. In such a case, a slight fluctuation in parameter $k_{l}$ will be observed according to Equation 16. This fluctuation will be manifested as a fluctuation of the electric charge according to Equation 7 and it may be observed for distances $r$ of cosmologic scale. For smaller scale distances, the fluctuation of the electric charge cannot be observed, due to the extremely slow rate with which the selfvariation of the electric charge evolves. We note that a corresponding fluctuation cannot occur for the selfvariation of the rest mass. Where the potential $V_{0}$ appears in Equation 2, the constant factor $c^{2}$ appears in Equation 1.

By the very slight variation of the fine structure parameter (Webb et al., 2011) we conclude that parameter $k_{l}$ has an extremely small value and by approximating:

$$
\exp \left(-\frac{k_{1} r}{c}\right)=1-\frac{k_{1} r}{c}
$$

In Equation 7, we obtain:

$$
\begin{aligned}
& \frac{q(r)}{q}=\frac{1-B}{1-B\left(1-\frac{k_{1} r}{c}\right)} \\
& \frac{q(r)}{q}=\frac{1-B}{1-B+k_{1} B \frac{r}{c}} \frac{q(r)}{q}=\frac{1}{1+\frac{k_{1} B}{1-B} \frac{r}{c}}
\end{aligned}
$$

Denoting: 
$\frac{\alpha(r)}{\alpha}-1=-2 W \frac{r}{c} \frac{\alpha(r)-\alpha}{\alpha}=-2 W \frac{r}{c} \frac{\Delta \alpha}{\alpha}=-2 W \frac{r}{c}$

We now compare Equation 26 with the equation we have from the observational data (Webb et al., 2011):

$\frac{\Delta \alpha}{\alpha}=-(3.587 \pm 0.815) \times 10^{-9} \mathrm{r} \cos \Theta$

In this equation the distance $r$ is measured in $M p c$ and not in GLyr. Since $1 M p c=3.261 \times 10^{6} \mathrm{Lyr}$, the quantity $(1.1 \pm 0.25) \quad$ (Webb et al., 2011) becomes $(3.587 \pm 0.815) \times 10^{-9}$ in Equation 27 .

According to Equation 27 the maximum decrease of the fine structure parameter is:

$$
\frac{\Delta \alpha}{\alpha}=-(3.587 \pm 0.815) \times 10^{-9} r
$$

For every specific distance $r$.

In Equation 28 we have the distance $r$ and not the redshift $z$. If we use the relativistic equation:

$r=\frac{c}{H} \frac{(1+z)^{2}-1}{(1+z)^{2}+1}$

For $c=3 \times 10^{5} \frac{\mathrm{km}}{\mathrm{s}}, H=68 \frac{\mathrm{km}}{\mathrm{sMpc}}$ and $\mathrm{z}=4$, we get:

$r=4072 M p c$

Substituting this distance into Equation 28 we obtain Equation 31:

$$
\frac{\Delta \alpha}{\alpha} \sim-1.46 \times 10^{-5}
$$

Which is of the order of magnitude where quantity $\frac{\Delta \alpha}{\alpha}$ is measured. But in the model of the selfvariations (Manousos, 2013a), the distance $r$ is given as a function of the redshift $z$ by Equation:

$$
\begin{aligned}
& r=\frac{c}{H} \frac{A}{1-A} \ln \left(\frac{A}{A-z(1-A)}\right) \\
& \quad \text { For } c=3 \times 10^{5} \frac{\mathrm{km}}{\mathrm{s}}, H=68 \frac{\mathrm{km}}{\mathrm{sMpc}}, A=0.999 \text { and } z=4,
\end{aligned}
$$

Equation 32 gives: $r=17652 M p c$

This distance is much greater than the one given by Equation 30. Since in the model of selfvariations we use Equation 32, we have to correct the numeric coefficients in Equation 27 and 28. If the quantity $\frac{\Delta \alpha}{\alpha}$ had been expressed as a function of the redshift $\mathrm{z}$ and not as a function of the distance $r$ in Equation 27, this correction would not have been necessary.

Taking into account Equation 30 and 33, we correct the coefficients of Equation 27 and 28 by multiplying with the ratio $\frac{4072}{17652}$, in order to use Equation 32 and not Equation 29. Thus, we obtain the following equation, corresponding to Equation 28:

$\frac{\Delta \alpha}{\alpha}=-(8.27 \pm 1.99) \times 10^{-10} r$

Similarly, we obtain the following equation, corresponding to Equation 27:

$\frac{\Delta \alpha}{\alpha}=-(8.27 \pm 1.99) \times 10^{-10} r \cos \Theta$

Using the pair of Equation 32 and 35, which is in agreement with the model of the selfvariations, we see that quantity $\frac{\Delta \alpha}{\alpha}$ increases from values of order $10^{-6}$ for small distances, up to values of order $5 \times 10^{-5}$ for larger distances. For $z=2$ we get $r=8818 M p c$ and $\frac{\Delta \alpha}{\alpha}=-0.73 \times 10^{-5}=-7.3 \times 10^{-6}$, for $z=7$ we get $r=$ $3039 M p c$ and $\frac{\Delta \alpha}{\alpha}=-2.56 \times 10^{-5}$, whereas for $z=12$ we get $r=53173 M p c$ and $\frac{\Delta \alpha}{\alpha}=-4.40 \times 10^{-5}$. This smooth increase is not observed when we use the pair of Equation 29 and 27. For $z=2$ we have $r=3529 \mathrm{Mpc}$ and $\frac{\Delta \alpha}{\alpha}=-1.26 \times 10^{-5}$, for $z=7$ we have $r=4275 \mathrm{Mpc}$ and $\frac{\Delta \alpha}{\alpha}=-1.53 \times 10^{-5}$ and for $z=12$ we get $r=4366 \mathrm{Mpc}$ and $\frac{\Delta \alpha}{\alpha}=-1.56 \times 10^{-5}$. A re-evaluation of the available data on the variation of parameter $\alpha$ (Webb et al., 2011) using the correct distances of distant astronomical objects as 
given by Equation 32, is required. The ratio $\frac{\Delta \alpha}{\alpha}$ depends on the distance $r$ of the astronomical object, but this cannot be expressed as long as we use the erroneous, smaller than the actual, distances of the standard cosmological model. We note that an analogous problem has arisen with the large luminosity distances of Type Ia supernovae (Riess et al., 1988; Perlmutter et al., 1999). A problem that goes away if we take into account the cosmological model of the selfvariations (Manousos, 2013a; 2013b).

Comparing Equation 34 and 26 we get:

$$
\frac{2 W}{c}=(8.27 \pm 1.99) \times 10^{-10} \frac{1}{M p c}
$$

And since $c=3 \times 10^{5} \frac{\mathrm{km}}{\mathrm{s}}$ we get:

$W=(1.24 \pm 0.30) \times 10^{-4} \frac{\mathrm{km}}{\mathrm{sMpc}}$

The quantity $W=\frac{k_{1} B}{1-B}$ expresses for the selfvariation of the electric charge, what Hubble's parameter $H=\frac{k A}{1-A}$ expresses for the selfvariation of the rest mass. From Equation 36 and for $H=68 \frac{\mathrm{km}}{s M p c}$, we obtain Equation 37:

$\frac{W}{H} \sim 1.8 \times 10^{-6}$

Relation (37) shows an extremely slow rate of evolution of the selfvariation of the electric charge, compared to the rate of evolution of the selfvariation of the rest mass.

In previous articles about the cosmological model of the selfvariations, we have used the value $H=60 \frac{\mathrm{km}}{\mathrm{sMpc}}$ for Hubble's parameter instead of the value $H=68 \frac{\mathrm{km}}{\mathrm{sMpc}}$ measured by the Planck satellite. The estimation $H=60 \frac{\mathrm{km}}{s M p c}$ was made at a time when the Hubble parameter was given around $72 \frac{\mathrm{km}}{s M p c}$, for specific reasons (Manousos, 2013a). For the same reasons we insist that the value of Hubble's parameter will drop below $68 \frac{\mathrm{km}}{s M p c}$ as the observational instruments improve and will stabilize around $60 \frac{\mathrm{km}}{s M p c}$. In any case, the predictions of the model of the selfvariations are not affected by the choice of one or the other value for Hubble's parameter. Only a slight modification of the numerical values predicted by the model results.

If we repeat the process with which we arrived at Equation 27 and 28 to Equation 34 and 35, using the Hubble parameter $H=60 \frac{\mathrm{km}}{s M p c}$, Equation 34 and 35 are written as Equation 38 and 39:

$\frac{\Delta \alpha}{\alpha}=-(8.07 \pm 1.93) \times 10^{-10} r$

$\frac{\Delta \alpha}{\alpha}=-(8.07 \pm 1.93) \times 10^{-10} r \cos \Theta$

Similarly, Equation 36 can be written as Equation 40:

$W=(1.21 \pm 0.29) \times 10^{-4} \frac{\mathrm{km}}{\mathrm{sMpc}}$

In order to calculate the exact value of parameter $\mathrm{W}$, as well as the arithmetic values of the preceding Equations, the re-evaluation of the observational data (Webb et al., 2011) on the basis of the model of the selfvariations is required. Of course, we expect the next generation of observational instruments to contribute to the accurate measurement of the fundamental parameter W.

Taking into account the dependence of the fine structure parameter on the angle $\Theta$, according to Equation 35, Equation 22 is written:

$\frac{q(z)}{q}=\frac{q(r)}{q}=1-\frac{\mathrm{Wr}}{\mathrm{c}} \cos \Theta$

The fluctuation of parameter $k_{l}$ in Equation 19 implies the fluctuation of parameter $W$, as well. Furthermore, the Milky Way is located at a random position in the Universe and, therefore, there are regions at which we observe a smaller value of parameter $\alpha(r)$ than the laboratory value $\left(\frac{\Delta \alpha}{\alpha}<0\right)$ and regions in which we observe a greater value of parameter $\alpha(r)$ than the 
laboratory value $\left(\frac{\Delta \alpha}{\alpha}>0\right)$. This fact, together with the fluctuation of parameter $\mathrm{W}$, is expressed by the term $\cos \theta$ in Equation 35 and 41.

We will now make two remarks as counterarguments to the views expressed about a privileged position of the Milky Way in the Universe, or about privileged directions in the Universe. Views that question the validity of Einstein's Relativity Principle.

The symmetric interval $\left[-5 \times 10^{-5},+5 \times 10^{-5}\right]$ in which the ratio $\frac{\Delta \alpha}{\alpha}$ belongs (Webb et al., 2011) expresses the inability of our current observational instruments to measure the variation of parameter $\alpha$ at larger distances, where this variation will be greater $\left(\frac{\Delta \alpha}{\alpha}>5 \times 10^{-5}\right)$. If we improve the observational instruments, we predict that the interval in which the ratio $\frac{\Delta \alpha}{\alpha}$ varies will not be symmetric with respect to zero, as a consequence of the random position of the Milky Way in the Universe.

The second remark concerns the angle $\Theta$ of Equation 35. Assuming a coordinate system $r, \theta, \varphi$ centered on the Milky Way and since the Milky Way occupies a random position in the Universe, it will be Equation 42:

$\Theta=\Theta(r, \theta, \varphi)$

That is, there will not be an equal distribution between the regions of the Universe where $\frac{\Delta \alpha}{\alpha}<0$ and the regions where $\frac{\Delta \alpha}{\alpha}>0$. To be more precise, what must be done is a detailed study about the anisotropies predicted by the cosmological model of the selfvariations. We stress again that the main reason for the anisotropies recorded by the observational instruments is the fact that we only observe a small part of the Universe (Manousos 2013a; 2013b). The isotropy of the Universe is expected at much larger scales, at much greater distances than the ones we observe today.

\section{THE CONTRIBUTION OF THE SELFVARIATION OF THE ELECTRIC CHARGE TO THE REDSHIFT}

The contribution of the selfvariation of the electric charge, to the redshift of distant astronomical objects is small, because of the slow rate of its evolution.
Nevertheless, this contribution could be detected in high accuracy cosmological measurements. In this paragraph we calculate this contribution.

Taking into account relation (6) we calculate the limit value of the ratio $\frac{m_{0}(r)}{m_{0}}$ for $A \rightarrow 1^{-}$. From Equation 4 we get:

$$
k=\frac{H(1-A)}{A}
$$

And write Equation 3 in the form:

$$
\frac{m_{0}(r)}{m_{0}}=\frac{1-A}{1-A \exp \left(-\frac{H(1-A)}{A} \frac{r}{c}\right)}
$$

Then, from Equation 43 easily follows that for $\mathrm{A} \rightarrow 1^{-}$it is:

$$
\frac{m_{0}(r)}{m_{0}}=\frac{1}{1+\frac{H r}{c}}
$$

We now get from Equation 41:

$$
\left(\frac{q(r)}{q}\right)^{4}=\left(1-\frac{W r}{c} \cos \Theta\right)^{4}
$$

And because of the small value of $\frac{W}{c} \sim 10^{-10}$, we make the approximation:

$\left(\frac{q(r)}{q}\right)^{4}=1-4 \frac{W r}{c} \cos \Theta$

We now get:

$z=\frac{\lambda-\lambda_{0}}{\lambda_{0}}=\frac{\lambda}{\lambda_{0}}-1 \quad z=\frac{X_{n}}{X_{n}(r)}-1$

The atomic excitation energy $X_{n}$ is proportional to the factor $m_{0} q^{4}$, where $m_{0}$ the rest mass and $q$ the electric charge of the electron and, therefore, Equation 46 can be written as:

$$
z=\frac{m_{0}}{m_{0}(r)}\left(\frac{q}{q(r)}\right)^{4}-1
$$


And with Equation 44 and 45 we obtain:

$$
\begin{aligned}
& z=\frac{1+\frac{H r}{c}}{1-4 \frac{W r}{c} \cos \Theta}-1 \\
& z=\frac{\frac{H r}{c}+4 \frac{W r}{c} \cos \Theta}{1-4 \frac{W r}{c} \cos \Theta} z=\frac{H r}{c} \cdot \frac{1+4 \frac{W}{H} \cos \Theta}{1-4 \frac{W r}{c} \cos \Theta}
\end{aligned}
$$

From Equation 47, solving for the distance $r$ of the astronomical object, we obtain:

$$
r=\frac{c}{H} \cdot \frac{z}{1+\frac{4 W}{H}(1+z) \cos \Theta}
$$

Because of the way Equation 47 and 48 have been derived, they only hold for the visible Universe. For greater distances Equation 7 and not Equation 41 and 35, should be used to determine the consequences of the selfvariation of the electric charge.

Combining Equation 44 and 48 we get:

$$
\frac{m_{0}(r)}{m_{0}}=\frac{1+\frac{4 W}{H}(1+z) \cos \Theta}{(1+z)\left(1+\frac{4 W}{H} \cos \Theta\right)}
$$

Taking into account that $\frac{W}{H} \sim 10^{-6}$, we derive from Equation 49 equation:

$$
\frac{m_{0}(r)}{m_{0}}=\frac{1}{(1+z)}
$$

Which results if we ignore the selfvariation of the electric charge (Manousos, 2013a).

From Equation 49 we obtain equation:

$$
\frac{\Delta m_{0}(r) c^{2}}{\Delta m_{0} c^{2}}=\frac{1+\frac{4 W}{H}(1+z) \cos \Theta}{(1+z)\left(1+\frac{4 W}{H} \cos \Theta\right)}
$$

During the conversion of rest mass $\Delta m_{0}$ into energy $\Delta m_{0} c^{2}$.

\section{ON THE TEMPERATURE FLUCTUATION OF THE COSMIC MICROWAVE BACKGROUND RADIATION}

The selfvariations affect almost all astrophysical parameters. In this and the next two paragraphs, we will see how the selfvariations affect the temperature of distant astronomical objects. As an aside, a temperature fluctuation of the CMBR, of the order of the fourth or fifth decimal place, emerges.

We consider a system of $\mathrm{N}$ particles, which is provided with energy through the process of conversion of rest mass into energy. For the laboratory we get equation:

$$
\Delta m_{0} c^{2}=N \frac{3}{2} K T
$$

While for a distant astronomical object the same equation is written:

$$
\Delta m_{0}(z) c^{2}=N \frac{3}{2} K T(z)
$$

Combining the above equations we obtain:

$$
\frac{T(z)}{T}=\frac{\Delta m_{0}(z) c^{2}}{\Delta m_{0} c^{2}}
$$

And with Equation 50 we see that:

$$
\frac{T(z)}{T}=\frac{1+\frac{4 W}{H}(1+z) \cos \Theta}{(1+z)\left(1+\frac{4 W}{H} \cos \Theta\right)}
$$

Equation 51 gives the temperature $T(z)$ of a distant astronomical object compared to the expected temperature $\mathrm{T}$, in the case of an object powered by the conversion of rest mass into energy.

The very early Universe predicted by the law of selfvariations, only slightly differs from the vacuum at a temperature close to $0 K$. Starting from this initial state of the Universe, the first energy conversions came from changes that occurred at the level particles, long before the gravitational accumulation of matter began. Therefore, the energy of material particles originated by the conversion of rest mass into energy during the formation and evolution of the primordial particles. 
Thus, we conclude that between the real temperature $T(z)$ of the CMBR and the measured temperature $\mathrm{T}$, Equation 51 holds. Considering the effect of the redshift $z$, we correct the energy of the CMBR photons by removing the consequences of the redshift. This correction in Planck's law regarding a black body, is equivalent with equation:

$$
T(z)(1+z)=T_{0}=2.726 K
$$

From Equation 52 we see that:

$$
T(z)=\frac{T_{0}}{(1+z)}
$$

And substituting $T(z)$ into Equation 51 we get:

$$
\frac{T_{0}}{T}=\frac{1+\frac{4 W}{H}(1+z) \cos \Theta}{1+\frac{4 W}{H} \cos \Theta}
$$

And finally:

$$
T=T_{0} \frac{1+\frac{4 W}{H} \cos \Theta}{1+\frac{4 W}{H}(1+z) \cos \Theta}
$$

Equation 53 predicts a fluctuation of the measured temperature $\mathrm{T}$ because of the fluctuation of the electric charge, compared to the constant temperature $\mathrm{T}_{0}$ of Equation 52 for the elimination of the consequences of the redshift on the CMBR.

We estimate the difference $\Delta T=T-T_{0}$ based on Equation 53. After performing the necessary calculations, we get:

$$
\Delta T=T-T_{0}=-T_{0} \frac{\frac{4 W z}{H} \cos \Theta}{1+\frac{4 W}{H}(1+z) \cos \Theta}
$$

Taking into account that $\frac{W}{H} \sim 1,8 \cdot 10^{-6}$, Equation (54) can be approximated as Equation 55:

$$
\Delta T=-T_{0} \frac{4 W z}{H} \cos \Theta
$$

Considering that $\frac{W}{H} \sim 1,8 \cdot 10^{-6}, T_{0}=2.726 \mathrm{~K}$, the value of the redshift $z$ at the boundaries of the observable Universe, where the CMBR originated and that $-1 \leq \cos \Theta \leq 1$, a fluctuation of the temperature of the CMBR results at the fourth or fifth decimal place (Hinshaw et al., 2009).

\section{THE RELATION BETWEEN TEMPERATURES T(Z) AND T IN THE CASE OF THE GRAVITATIONAL ACCUMULATION OF MATTER}

In the case when a system of $\mathrm{N}$ particles of total mass $\mathrm{M}$ acquires its energy by the gravitational accumulation of matter, we have:

$\frac{3 G M^{2}}{5 R}=N \frac{3}{2} K T$

For the laboratory and:

$\frac{3 G M^{2}(z)}{5 R}=N \frac{3}{2} K T(z)$

For the distant astronomical object. From the previous Equations we obtain:

$$
\frac{T(z)}{T}=\frac{M^{2}(z)}{M^{2}}
$$

And taking into account Equation 49 we arrive at relation:

$\frac{T(z)}{T}=\left[\frac{1+\frac{4 W}{H}(1+z) \cos \Theta}{(1+z)\left(1+\frac{4 W}{H} \cos \Theta\right)}\right]^{2}$

Equation 58 gives the real temperature $T(z)$ in relation to the expected temperature $T$, for a distant astronomical object powered by the gravitational collapse of matter. Considering that $\frac{W}{H} \sim 1,8 \cdot 10^{-6}$, Equation 58 gives the approximation:

$$
\frac{T(z)}{T}=\frac{1}{(1+z)^{2}}
$$

Equation 58 should be used instead of Equation 59, in the case of high accuracy measurements. In that case, 
however, a possible difference in the distance $R$ between Equation 56 and 57, should also be taken into account. Furthermore, one should consider that the redshift affects the degree of atomic ionization and, therefore, the multitude $N$ of particles appearing in Equation 57 and also the opacity of stellar surfaces (Manousos, 2013b).

\section{THE SELFVARIATIONS DO NOT SIGNIFICANTLY AFFECT THE FUSION TEMPERATURE OF HYDROGEN}

The fusion temperature $T(z)$ of hydrogen at distant astronomical objects is practically equal to the laboratory value $T$, because of the extremely slow rate of evolution of the selfvariation of the electric charge. During fusion, the thermal energy $\frac{3}{2} K T$ of the protons balances the potential energy due to their mutual repulsion, $\frac{1}{4 \pi \varepsilon_{0}} \frac{q^{2}}{d}$, so that Equation 60 :

$$
\frac{1}{4 \pi \varepsilon_{0}} \frac{q^{2}}{d}=\frac{3}{2} K T
$$

The same Equation at a distant astronomical object is written Equation 61:

$$
\frac{1}{4 \pi \varepsilon_{0}} \frac{q^{2}(z)}{d}=\frac{3}{2} K T(r)
$$

If we assume that the distance $d$ is not affected by the selfvariations, something very likely, we get by the above Equations:

$$
\frac{T(r)}{T}=\left(\frac{q(r)}{q}\right)^{2}
$$

Combining Equation 41 and 62 we get:

$$
\frac{T(r)}{T}=\left(1-\frac{W r}{c} \cos \Theta\right)^{2}
$$

Combining Equation 63 and 48, we get after the calculations:

$$
\frac{T(r)}{T}=\frac{T(z)}{T}=\left[\frac{1+\frac{W}{H}(4+3 z) \cos \Theta}{1+\frac{4 W}{H}(1+z) \cos \Theta}\right]^{2}
$$

Since $\frac{W}{H} \sim 1,8 \cdot 10^{-6}$, we conclude that $\mathrm{T}(\mathrm{z}) \sim \mathrm{T}$. Therefore, the fusion temperature of hydrogen is not affected by the selfvariations in the region of the observable Universe. The general Equation is:

$$
\frac{T(r)}{T}=\left(\frac{1-B}{1-B \exp \left(-\frac{k_{1} r}{c}\right)}\right)^{2}
$$

Which it is obtained by combining Equation 62 and 7.

\section{ON THE TEMPERATURE DIFFERENCE BETWEEN THE NORTHERN AND SOUTHERN HEMISPHERE OF THE UNIVERSE}

We write Equation 35 in the form:

$$
\frac{\Delta \alpha}{\alpha}=-\frac{2 \mathrm{~W}}{\mathrm{c}} \mathrm{r} \cos \Theta
$$

Combining Equation 65 and 48 we obtain:

$$
\frac{\Delta \alpha}{\alpha}=-\frac{\frac{2 W}{H} z \cos \Theta}{1+\frac{4 W}{H}(1+z) \cos \Theta}
$$

Combining Equation 66 and 54 we obtain:

$$
\frac{\Delta \alpha}{\alpha}=\frac{1}{2} \frac{\Delta T}{T_{0}}
$$

Equation 67 has the advantage that it is independent of the parameters $\mathrm{H}, \mathrm{W}$ and $\cos \Theta$. It also allows the calculation of the ratio $\frac{\Delta \alpha}{\alpha}$ for very large values of the redshift $\mathrm{z}$, at the boundaries of the observable Universe, as a function of the ratio $\frac{\Delta T}{T_{0}}$.

For the Northern hemisphere of the Universe we know (King et al., 2011; Webb et al., 2011) that on average $\Delta \alpha<0$, so from Equation 67 we get Equation 68:

$T<T_{0}$

For the Southern hemisphere we know that on average $\Delta \alpha>0$, therefore Equation 69: 


$$
T>T_{0}
$$

The temperature difference between the Northern and the Southern hemisphere of the Universe is a consequence of the selfvariation of the electric charge. The slight fluctuation of the electric charge in the various regions of the observable Universe have as a consequence a corresponding slight fluctuation of the temperature, with smaller electric charge in the past, corresponding to lower temperatures. We also come to the same conclusion from the dependence of the Thomson $\kappa \alpha$ Klein-Nishina scattering coefficients, as well as the degree of atomic ionization, on the electric charge of the electron. In the regions of the Universe with slightly smaller electric charge in the past, slightly lower temperatures are predicted compared to regions of the Universe where the electric charge had slightly larger value. We shall not present these arguments in the current article, but all analyses lead to the same conclusion about how the selfvariation of the electric charge affects the temperature of the Universe.

\section{ON THE OKLO NATURAL NUCLEAR REACTOR}

In Equation 41, factor $\cos \Theta$ expresses the fluctuation of the constant $\mathrm{k}_{1}$ and the random position of the Milky Way in the Universe. There are regions of the Universe where parameter $\alpha$ is slightly smaller than the laboratory value $(\cos \Theta>0)$ and parts where it is slightly larger $(\cos \Theta<0)$. For phenomena that occur on earth, like the Oklo natural nuclear reactor, the consequences of the increase of the electric charge with the passage of time dominate. For the description of such phenomena, we use Equation 26.

Before a time interval $\frac{r}{c}=t$ from "now", Equation 26 gives:

$$
\frac{\Delta \alpha}{\alpha}=-2 W t
$$

Considering that $W=1.24 \times 10^{-4} \frac{\mathrm{km}}{\mathrm{sMpc}}, 1 M p c=$ $3.086 \times 10^{19} \mathrm{~km}$ and $1 y r=3.153 \times 10^{7} \mathrm{~s}$, Equation 70 is written Equation 71:

$$
\frac{\Delta \alpha}{\alpha}=-2.52 \times 10^{-16} t
$$

where, the time interval $t$ is measured in year.
For $t=2 \times 10^{9} \mathrm{yr}$, a time interval of the order of magnitude of the operation of the Oklo natural nuclear reactor, we get:

$$
\frac{\Delta \alpha}{\alpha}=-5 \times 10^{-7}
$$

This variation is extremely small and it is difficult to measure (Petrov et al., 2006; Meshik et al., 2004; Gauthier-Lafaye, 2002; De Laeter et al., 1980). We expect that the processing of the cosmological data we possess, as well as the improvement of the observational instruments, will give us a more accurate value for the fundamental parameter $\mathrm{W}$. However, this more accurate measurement cannot considerably affect the theoretical prediction about Oklo's reactor, since Equation 72 gives an extremely small value for the ratio $\frac{\Delta \alpha}{\alpha}$.

\section{RESULTS}

We summarize our obtained results.

We predict the absence of antimatter in the Universe as a consequence of the ability of the electric potential $\mathrm{V}_{0}$ to be either positive or negative in Equation 16, $k_{1}=\frac{b V_{0} \sigma_{1}}{\hbar}$. The antimatter particles lose their electric charge over time and are converted into electrically neutral particles. A part of Dark Matter is obtained by this mechanism.

We provide for the variation of the fine structure 'constant' $\alpha$ by Equation $35 \frac{\Delta \alpha}{\alpha}=-\frac{2 W}{c} r \cos \Theta$.

We ccalculate the contribution of the selfvariation of the electric charge to the redshift $z$ of astronomical objects through the Equation 47 and 48, $z=\frac{H r}{c} \cdot \frac{1+4 \frac{W}{H} \cos \Theta}{1-4 \frac{W r}{c} \cos \Theta} r=\frac{c}{H} \cdot \frac{z}{1+\frac{4 W}{H}(1+z) \cos \Theta}$.

We give the reason for the fluctuation in the temperature of the CMBR by Equation 55, $\Delta T=-T_{0} \frac{4 W z}{H} \cos \Theta$.

The Equation 51, $\frac{T(z)}{T}=\frac{1+\frac{4 W}{H}(1+z) \cos \Theta}{(1+z)\left(1+\frac{4 W}{H} \cos \Theta\right)}$ gives

the temperature $T(z)$ acquired by a distant astronomical object when converting rest mass into energy, compared 
with the temperature $\mathrm{T}$ acquired by the same object with the same mechanism in our galaxy.

The Equation 58, $\frac{T(z)}{T}=\left[\frac{1+\frac{4 W}{H}(1+z) \cos \Theta}{(1+z)\left(1+\frac{4 W}{H} \cos \Theta\right)}\right]^{2}$ gives the temperature $T(z)$ acquired by a distant astronomical object due to gravitational aggregation of matter, compared with the temperature acquired by the same object with the same mechanism in our galaxy.

The Equation 64, $\frac{T(r)}{T}=\frac{T(z)}{T}=\left[\frac{1+\frac{W}{H}(4+3 z) \cos \Theta}{1+\frac{4 W}{H}(1+z) \cos \Theta}\right]^{2}$ gives the fusion temperature of hydrogen $T(z)$ as a function of redshift $\mathrm{z}$ within the limits of the observable Universe, compared to the laboratory temperature $\mathrm{T} \sim 2 \times 10^{8} \mathrm{~K}$. The general equation valid for any distance $\mathrm{r}$ is $\frac{T(r)}{T}=\left(\frac{1-B}{1-B \exp \left(-\frac{k_{1} r}{c}\right)}\right)^{2}$.

The Equation 67, $\frac{\Delta \alpha}{\alpha}=\frac{1}{2} \frac{\Delta T}{T_{0}}$ relates the variation $\frac{\Delta \alpha}{\alpha}$ of the fine structure 'constant' at the limits of the observable Universe with the fluctuation $\frac{\Delta T}{T_{0}}$ of the temperature of the CMBR.

The Equation 70, $\frac{\Delta \alpha}{\alpha}=-2 W t$ gives the change of the fine structure 'constant' before a time period $t$, for measurements conducted on earth.

\section{DISCUSSION}

The potential for the electric charge to evolve in two directions, constitutes a pronounced anisotropy in the macrocosm. Among the first consequences is the absence of antimatter from the Universe today. Since the electric charge appears in the Universe as pairs of opposite physical quantities and it does not have to start from a zero initial value, the potential for the evolution of the electric charge in two directions exists, even if we could not determine the exact cause, i.e., the change in sign of the electromagnetic potential $\mathrm{V}_{0}$. But the law of selfvariations, that quantitatively determines this phenomenon, is compatible with special relativity and the lorentz-einstein transformations. The anisotropy in the macrocosm, due to the absence of antimatter, is apparent and not real.

During the evaluation of the observational data on the fluctuation of the fine structure parameter, the same problem appeared as in the evaluation of the luminosity distances of Type Ia supernovae. The distances of astronomical objects, especially for large values of the redshift, are much greater than those predicted by the standard cosmological model. If we take into account the predictions of the model of the selfvariations, the luminosity distances of Type Ia supernovae are completely justified. Furthermore, it emerges that the fluctuation of the fine structure parameter depends on the distance at which we measure it. This information is lost from the evaluation of the observational data, if we consider the predictions of the standard cosmological model.

The fluctuation of the electric charge justifies both the temperature fluctuation of the CMBR and the temperature difference between the two hemispheres of the Universe. The selfvariations affect almost the totality of parameters in Astrophysics. This fact requires an overall re-evaluation of the observational data we possess.

This article is part of a general study which provides a unified cause for the quantum phenomena and the cosmological data. The general study converges to the law of selfvariations. The law of the selfvariations contains enough information to justify the totality of the current cosmological data. The selfvariation of the rest mass is realized at an extremely slow pace. For this reason, the direct consequences of the selfvariation of the rest mass are recorded at cosmological distances. The selfvariation of the electric charge is realized at an even slower pace and its consequences are only recorded in high-precision measurements. The present day ultrasensitive observation instruments have the required precision and therefore the available cosmological data are also affected by the consequences of the selfvariation of the electric charge. This is exactly the reason why the standard cosmological model cannot justify these particular cosmological data.

\section{CONCLUSION}

The conclusion we arrive at is that the selfvariation of the electric charge can justify in common, as a unifying cause, the absence from the Universe of antimatter, which with the passage of time is converted into Dark Matter, the fluctuation of the fine structure parameter, the temperature fluctuation of the cosmic microwave 
background radiation and the temperature difference between the Northern and Southern hemisphere of the Universe. The research presented in this article concerns the observable Universe. This is due to the approximation we did in Equation 7 using Equation 22. For observations concerning much larger distances Equation 3 and 7 should be used. This article together with the already published articles referenced in previous sections give us a large number of equations which arise from the law of selfvariations. These equations are sufficient to carry out a computer simulation of the Universe as predicted by the law of selfvariations. We suggest the realization of this study from colleagues who are experts in the subject.

\section{REFERENCES}

Chand, H., R. Srianand, P. Petitjean and B. Aracil, 2004. Probing the cosmological variation of the finestructure constant: Results based on VLT-UVES sample. Astron. Astrophys, 417: 853-871. DOI: 10.1051/0004-6361:20035701

De Laeter, J.R., K.J.R. Rosman and C.L. Smith, 1980. The Oklo natural reactor: Cumulative fission yields and retentivity of the symmetric mass region fission products. Earth Planetary Sci. Lett., 50: 238-246. DOI: 10.1016/0012-821X(80)90135-1

Dzuba, V.A., V.V. Flambaum and J.K. Webb, 1999. Space-time variation of physical constants and relativistic corrections in atoms. Phys. Rev. Lett., 82: 888-891. DOI: 10.1103/PhysRevLett.82.888

Gauthier-Lafaye, F., 2002. Two billion year old natural analogs for nuclear waste disposal: The natural nuclear fission reactors in Gabon (Africa). Comptes Rendus Physique, 3: 839-849. DOI: 10.1016/S16310705(02)01351-8

Hinshaw, G., J.L. Weiland, R.S. Hill, N. Odegard and D. Larson et al., 2009. Five-Year Wilkinson Microwave Anisotropy Probe (WMAP) Observations: Data processing, sky maps and basic results. Astrophys. J. Supp., 180: 225-245. DOI: 10.1088/0067-0049/180/2/225

King, J.A., M.T. Murphy, W. Ubachs and J.K. Webb, 2011. New constraint on cosmological variation of the proton-to-electron mass ratio from Q0528-250. Mon. Not. Roy. Astron. Soc., 417: 3010-3024. DOI: 10.1111/j.1365-2966.2011.19460.x
Manousos, E., 2013a. Cosmological data could have a microscopic, not macroscopic, cause. Am. J. Space Sci., 1: 10-21. DOI: 10.3844/ajssp.2013.10.21

Manousos, E., 2013b. Mass and charge selfvariation: A common underlying cause for quantum phenomena and cosmological data. Progress Phys., 9: 73-141.

Meshik, A.P., C.M. Hohenberg and O.V. Pravdivtseva, 2004. Record of cycling operation of the natural nuclear reactor in the Oklo/Okelobondo area in gabon. Phys. Rev. Lett., 93: 182-302. DOI: 10.1103/PhysRevLett.93.182302

Molaro, P., S.A. Levshakov, S. Monai, M. Centurion and P. Bonifacio et al., 2008. UVES radial velocity accuracy from asteroid observations. Implications for the fine structure constant variability. Astron. Astrophys, 481: 559-569. DOI: 10.1051/00046361:20078864

Murphy, M.T., P. Tzanavaris, J.K. Webb and C. Lovis, 2007. Selection of ThAr lines for wavelength calibration of echelle spectra and implications for variations in the fine-structure constant. Mon. Not. Roy. Astron. Soc., 378: 221-230. DOI: 10.1111/j.1365-2966.2007.11768.x

Murphy, M.T., J.K. Webb and V.V. Flambaum, 2003. Further evidence for a variable fine-structure constant from Keck/HIRES QSO absorption spectra. Mon. Not. Roy. Astron. Soc., 345: 609-609. DOI: 10.1046/j.1365-8711.2003.06970.x

Murphy, M.T., J.K. Webb and V.V. Flambaum, 2008. Revision of VLT/UVES constraints on a varying fine-structure constant. Mon. Not. Roy. Astron. Soc., 384: 1053-1062. DOI: 10.1111/j.13652966.2007.12695.x

Perlmutter, S., M.S. Turner and M. White, 1999. Constraining dark energy with Type Ia supernovae and Large-Scale structure. Phys. Rev. Lett., 83: 670-673. DOI: 10.1103/PhysRevLett.83.670

Petrov, Y.V., A.I. Nazarov, M.S. Onegin and E.G. Sakhnovsky, 2006. Natural nuclear reactor at Oklo and variation of fundamental constants: Computation of neutronics of a fresh core. Phys. Rev. C, 74: 664-610. DOI: 10.1103/PHYSREVC.74.064610

Riess, A.G., A.V. Filippenko, P. Challis, A. Clocchiatti and A. Diercks et al., 1998. Observational evidence from supernovae for an 
accelerating Universe and a cosmological constant. Astronomical. J., 116: 1009-1038. DOI: $10.1086 / 300499$

Tzanavaris, P., J.K. Webb, M.T. Murphy, V.V. Flambaum and S.J. Curran, 2005. Limits on variations in fundamental constants from $21-\mathrm{cm}$ and ultraviolet Quasar absorption lines. Phys. Rev. Lett., 95: 41301-41304. DOI: 10.1103/PhysRevLett.95.041301

Webb, J.K., M.T. Murphy, V.V. Flambaum, V.A. Dzuba and J.D. Barrow et al., 2001. Further evidence for cosmological evolution of the fine structure constant. Phys. Rev. Lett., 87: 291-301. DOI: 10.1103/PhysRevLett.87.091301
Webb, J.K., J.A. King, M.T. Murphy, V.V. Flambaum and R.F. Carswell et al., 2011. Indications of a spatial variation of the fine structure constant. Phys. Rev. Lett., DOI: 10.1103/PhysRevLett.107.191101

Webb, J.K., V.V. Flambaum, C.W. Churchill, M.J. Drinkwater and J.D. Barrow, 1999. Search for time variation of the fine structure constant. Phys. Rev. Lett., $\quad 82$ : 884-887. DOI: 10.1103/PhysRevLett.82.884 